\title{
Extended Triple-Junction Solar Cell 3D Distributed Model: Application to Chromatic Aberration-Related Losses
}

\author{
I. Garcia, P. Espinet-González, I. Rey-Stolle, E. Barrigón, and C. Algora \\ Instituto de Energía Solar-Universidad Politécnica de Madrid (IES-UPM) \\ E.T.S.I. Telecomunicación, Avda. Complutense 30, 28040 Madrid, Spain \\ igarcia@ies-def.upm.es・Tel: +34914533555•Fax: +34915446341
}

\begin{abstract}
An extended 3D distributed model based on distributed circuit units for the simulation of triple-junction solar cells under realistic conditions for the light distribution has been developed. A special emphasis has been put in the capability of the model to accurately account for current mismatch and chromatic aberration effects. This model has been validated, as shown by the good agreement between experimental and simulation results, for different light spot characteristics including spectral mismatch and irradiance non-uniformities. This model is then used for the prediction of the performance of a triple-junction solar cell for a light spot corresponding to a real optical architecture in order to illustrate its suitability in assisting concentrator system analysis and design process.
\end{abstract}

Keywords: triple-junction concentrator solar cell, distributed model, chromatic aberration.

PACS: $80.85 .30 . \mathrm{De}, 88.40 . \mathrm{fc}, 88.40 . j \mathrm{p}$

\section{INTRODUCTION}

Distributed effects in a solar cell consist on the existence of non-uniform voltage and current profiles across the area of the solar cell during the operation of the device. For example, when applying a non-uniform irradiance light profile to the solar cell, a distribution of photocurrents and voltages over the solar cell area appears, making that some regions of the solar cell work at a different voltage and current than that applied to the terminals of the whole solar cell device. Even more evident is the case of chromatic aberration in the light spot, which makes that distributed effects appear not only over the solar cell area but also between subcells in the multijunction solar cell. The design of the solar cell can also contribute to the enhancement of these distributed effects. For example, a high metal sheet resistance can give rise to an excessive voltage drop in the front grid that makes the inner part of the active area be at a higher voltage than the outer parts. Many more examples of distributed effects causes exist, having all of them in common a power loss behind.

Three-dimensional (3D) distributed models based on distributed circuit units have been demonstrated to be a convenient tool for the analysis of distributed effects and for the design of the solar cell device in order to minimize the impact of the distributed effects on the efficiency [1-4]. As compared to other modeling and simulation approaches, the computation resources needed are much less restrictive even for the simulation of large area solar cells [5].The Group of III-V Semiconductors at IES-UPM has a wide experience on this modeling approach. The first model published in 2005, for a GaAs single-junction solar cell [1], has been extended successively over the years, in order to be able to account for non-uniform irradiance distributions [3], to simulate dual-junction solar cells [6], to account for real tunnel-junction effects in a dual-junction solar cell [4], etc. In addition to the publications in journals derived from this extensive work, a patent application was filed concerning the model for real tunnel junction effects [7]. The evolution of our distributed model is closely linked to the development of the software package that allows the definition of the solar cell characteristics and the light spot properties and that is used to build the distributed models.

In this work, we present the first 3D distributed model based on distributed circuit units for a triplejunction solar cell, designed to simulate and predict the operation of the solar cell under real conditions. A special emphasis has been put in the design and development of the model to accurately account for real light spot characteristics, specially chromatic aberration.

\footnotetext{
7th International Conference on Concentrating Photovoltaic Systems

AIP Conf. Proc. 1407, 13-16 (2011); doi: 10.1063/1.3658284

(C) 2011 American Institute of Physics 978-0-7354-0979-8/\$30.00
} 


\section{TRIPLE-JUNCTION SOLAR CELL 3D DISTRIBUTED MODEL}

The general building procedure for the model consists, briefly, in the definition of the solar cell geometry, division of its area into sufficiently small sections, assignment of an equivalent circuit to each section (according to its characteristics), definition of the light spot characteristics and, finally, generation of the whole solar cell equivalent circuit by connecting all the sections through the lateral current conduction path resistors. Extended details on this procedure can be found elsewhere [1]. In the triple-junction solar cell model presented here, perimeter, metal-covered and exposed regions are distinguished in the solar cell. For each of these, the equivalent circuits we use are shown in figure 1. As compared to previous models developed, we have introduced an additional n-on-p junction for the bottom cell and the I-V characteristic description for the second tunnel junction of the triplejunction solar cell. Moreover, an extended definition of lateral current conduction paths has been implemented, which is key in accurately accounting for the effect of chromatic aberration in the light spot.

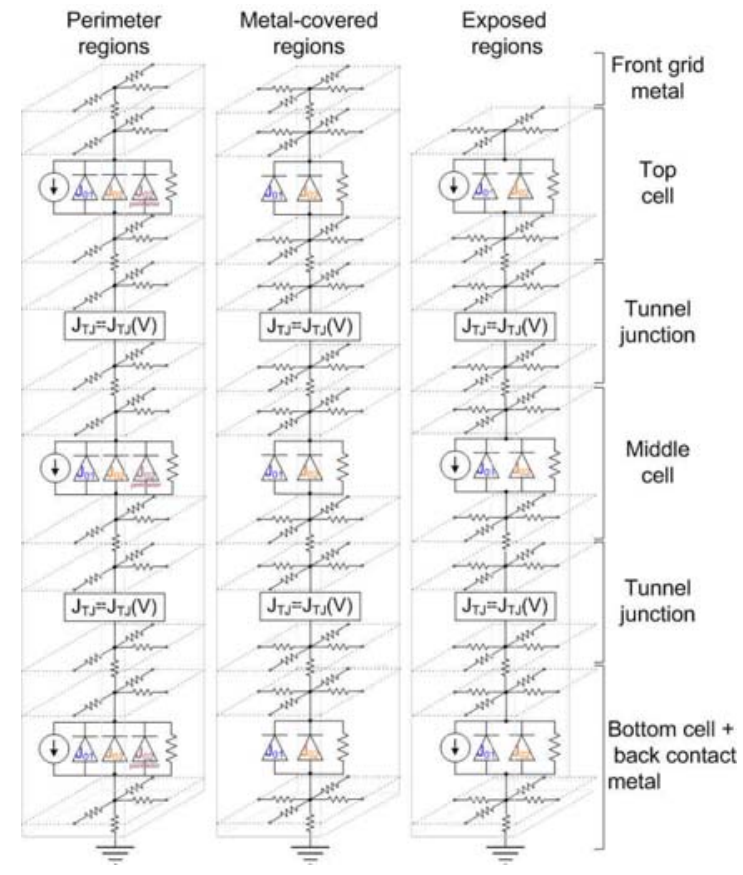

FIGURE 1. Equivalent circuit model for the three regions of the solar cell considered in the triple-junction model presented in this work.

\section{VALIDATION OF THE MODEL}

In order to analyze the ability to reproduce the operation of a triple-junction solar cell under real light spot conditions, the simulation results obtained using the distributed model described in last sections were compared to real characterization results. GaInP/GaInAs/Ge triple-junction concentrator solar cells fabricated at IES-UPM., whose parameters are well known by us, were used. For the experimental characterization, the flash lamp method was used. It is well known that the spectral composition of the light varies during the decay of a flash lamp pulse [8]. In figure 2-left, the irradiance of the light measured by a top, middle and bottom isotype reference cells during a flash pulse decay is shown. In order to characterize the response of the triple-junction solar cell for different spectral compositions in the light, I-V curves were measured at different times of the flash pulse decay. The 3D distributed model of these solar cells was generated and the light spot characteristics were reproduced. The comparison between the experimental and simulation short circuit current $\left(I_{s c}\right)$, open circuit voltage $\left(V_{o c}\right)$ and fill factor $(\mathrm{FF})$ data trends is shown in figure 3.

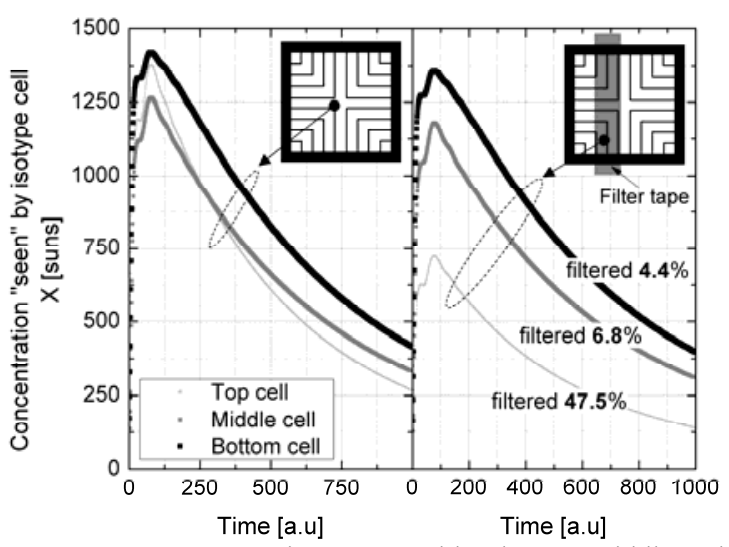

FIGURE 2. Concentration measured by the top, middle and bottom isotype cells during a flash pulse (left). Observe the spectral composition variation that takes place during the pulse decay. On the right, it is shown the composition of the flash light pulse after filtered by the tape used to create a spatial non-uniformity of the irradiance.

As can be seen, a good agreement between the experimental and simulation results is obtained. More significant than the quantitative agreement, which can be affected by the experimental characterization tolerances, is the good reproduction of the tendencies. Particularly revealing is the FF curve, where the concentration at which minimum FF is obtained at the current matching situation, is closely reproduced by the simulation. This means that the modeling is 
accurately accounting for the effects derived from spectral mismatch.

In order to introduce a spatial variation of irradiance and spectral composition, a filter tape was used to cover part of the solar cell area, as shown in the inset of figure 2-right. The spectral composition corresponding to the flash lamp pulse with this filter tape is shown in figure 2-right. Observe how the filter used attenuates mostly the light spectral range corresponding to the top cell. Again, the experimental measurements were compared to simulations carried out using the same illumination conditions. In figure 4 the results of this comparison are shown.

Again, a good agreement between experimental and simulation data is obtained. The eye-catching curve shift observed in the FF (though still being within $3.5 \%$ ) is possibly due to some inaccuracy in the determination of the recombination currents used in the model for the subcells. The $F F$ is quite sensitive to small variations in these recombination currents (even more than the $V_{o c}$ ) when current mismatch between subcells exists.

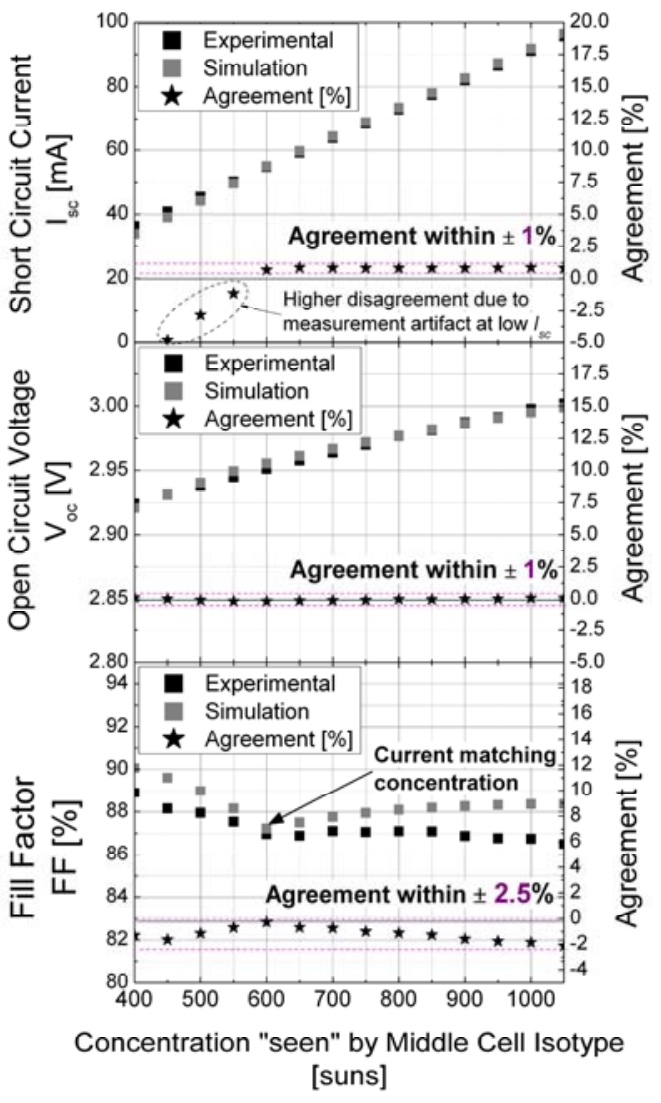

FIGURE 3. Measured and simulated short circuit current, open circuit voltage and fill factor plotted against the concentration measured by the middle cell isotype during the flash pulse.
In conclusion, the triple-junction solar cell distributed model developed accounts closely for the processes derived from a light spot with non-uniform irradiance distribution and chromatic aberration, with a good accuracy. Therefore, the model is validated and has been demonstrated to be predictive for simulation of the triple-junction solar cell under real light spot conditions.

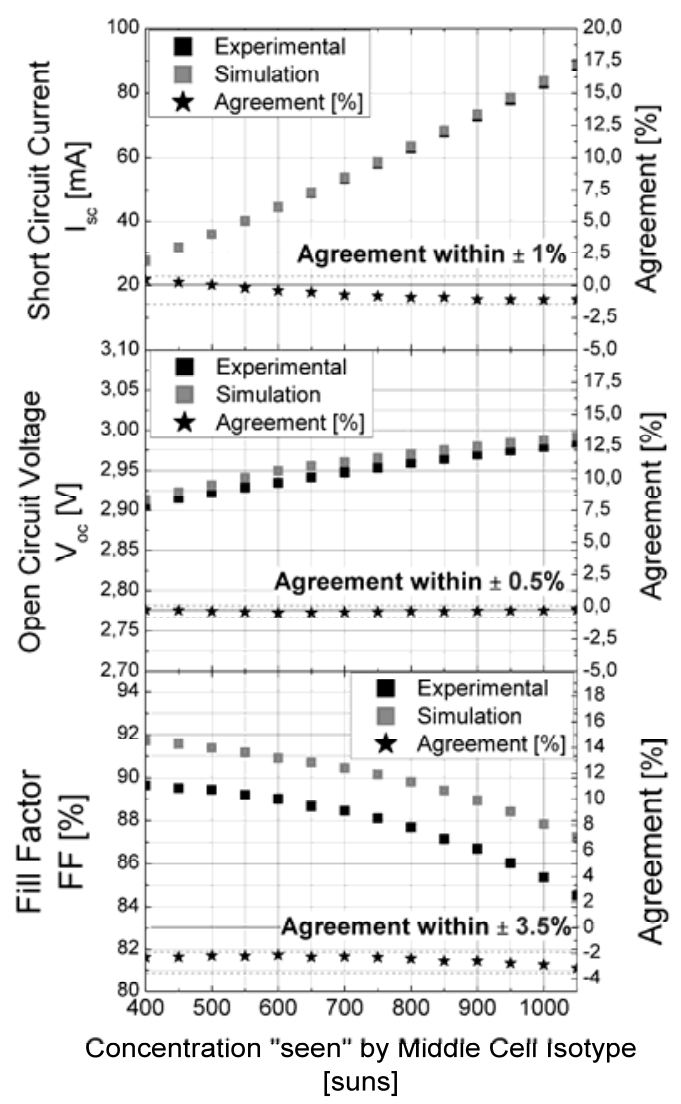

FIGURE 4. Measured and simulated short circuit current, open circuit voltage and fill factor for the cell with a filter tape covering part of its active area, plotted against the concentration measured by the isotype middle cell during the flash pulse.

\section{PREDICTION OF PERFORMANCE UNDER REAL CONDITIONS.}

Using the validated model, a $0.25 \mathrm{~cm}^{2}$ triple-junction solar cell I-V curve was simulated for a case with uniform light spot with 500 suns concentration and current matching. The I-V curve of the same solar cell device was then simulated using a light spot corresponding to an architecture of Fresnel lens + homogenizer, which exhibits chromatic aberration. 
The spatial and spectral composition of this light spot is illustrated in the inset of Figure 5.

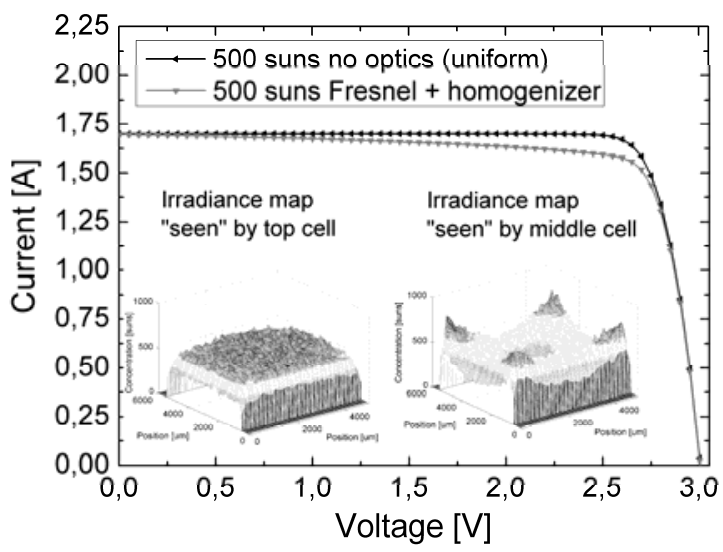

FIGURE 5. I-V curves of a $0.25 \mathrm{~cm}^{2}$ triple-junction solar cell illuminated with 500 suns of uniform light and spectral matching and the same curve obtained for a light spot exhibiting the irradiance maps shown in the inset.

The I-V curves obtained are shown in Figure 5. The effect of the Fresnel+homogenizer light spot consists on a parallel-resistance-like effect, where only the FF is affected. This effect is routinely observed in the characterization work carried out at IES-UPM's ISI group on this kind of systems. In the particular case simulated, the power loss generated by chromatic aberration is around 5\% relative. Of course, the effect of the chromatic aberration would be stronger for irradiance profiles with a higher aspect ratio. Moreover, it is also affected by the solar cell properties. For example, significant variations of the FF and $I_{s c}$ with the middle cell lateral resistance, for the light spot case simulated in Figure 5, have been observed. A detailed analysis of the influence of solar cell parameters on the effect of chromatic aberration is ongoing and will be presented in future works.

\section{SUMMARY AND CONCLUSIONS}

A 3D distributed model has been developed, which allows to simulate the performance of triple-junction solar cells under any light spot spectral composition and photocurrent distribution in each subcell. This model has been shown to accurately account for the effects produced by irradiance non-uniformities and chromatic aberration in the light spot, observed in practice. The strength of the modeling approach has been illustrated by the simulation of the I-V characteristic of a triple-junction solar cell illuminated with a real light spot exhibiting chromatic aberration. Consequently, this model is ready to assist in the design and optimization of the concentrator solar cell + optics ensemble, in order to maximize the efficiency of the concentrator system.

\section{ACKNOWLEDGMENTS}

The authors gratefully acknowledge the support of the ISI group at IES-UPM during flash lamp measurements and for useful discussions. This work has been funded by the Spanish Ministry of Science and Innovation by means of research projects with references TEC2008-01226 and TEC2009-11143, the CONSOLIDER-INGENIO 2010 program by means of the GENESIS FV project (CDS2006-004) and the project SIGMASOLES (PSE-440000-2009-8); and the Comunidad de Madrid under contract NUMANCIA II (S2009/ENE1477) and project CCG10-UPM/ENE566.

\section{REFERENCES}

1. B. Galiana, C. Algora, I. Rey-Stolle, I.García, “A 3-D model for concentrator solar cells based on distributed circuits units" IEEE Trans. Electron Devices $\mathbf{5 2}$ (12)(2005), pp. 2552-2558

2. M. Steiner, S.P. Philipps, M. Hermle, A. W. Bett, F. Dimroth, "Front contact grid optimization of III-V solar cells with spice network simulation" $24^{\text {th }}$ European Photovoltaic Solar Energy Conference, Hamburg, Germany, 2009.

3. I. García, C. Algora, I. Rey-Stolle and B. Galiana "Study of non-uniform ligt profiles on high concentration III-V solar cells using quasi 3D distributed models" $34^{\text {th }}$ IEEE Photovoltaic Specialist Conference, San Diego, California, USA, 2008

4. P. Espinet, I. García, I. Rey-Stolle, C. Algora and M. Baudrit, "Distributed simulaton of real tunnel junction effects in multi-junction solar cells" proceedings of the $C P V-6$ International Conference on Concentrating Photovoltaic Systems, Freiburg, Germany, 2010.

5. I. Rey-Stolle, C. Algora, I. García, M. Baudrit, P. Espinet, B. Galiana, E. Barrigón "Simulating III-V concentrator solar cells: a comparison of advantages and limitations of lumped analytical models, distributed analytical models and numerical simulations" in 35th IEEE Photovoltaic Specialist Conference, Philadelphia, USA, 2009.

6. I. García "Development of GaInP/GaAs dual-junction solar cells for high light concentrations". Thesis, Technical University of Madrid, 2010.

7. Universidad Politécnica de Madrid, "Method implemented in a computer for the numerical simulation of semiconductor devices containing funnel junctions" International Patent Application PCT/EP2011/055086.

8. C. Domínguez, I. Antón, and G. Sala, "Multijunction solar cell model for translating I-V characteristics as a function of irradiance, spectrum, and cell temperature," Progress in Photovoltaics: Research and Applications, vol. 18, pp. 272-284, 2011. 